\title{
Effects of Stimulation Rate, Mode and Level on Modulation Detection by Cochlear Implant Users
}

\author{
John J. Galvin III And QIAn-Jie Fu \\ Department of Auditory Implants and Perception, House Ear Institute, Los Angeles, CA 90057, USA
}

Received: 25 April 2005; Accepted: 6 June 2005; Online publication: 2 August 2005

\begin{abstract}
In cochlear implant (CI) patients, temporal processing is often poorest at low listening levels, making perception difficult for low-amplitude temporal cues that are important for consonant recognition and/or speech perception in noise. It remains unclear how speech processor parameters such as stimulation rate and stimulation mode may affect temporal processing, especially at low listening levels. The present study investigated the effects of these parameters on modulation detection by six CI users. Modulation detection thresholds (MDTs) were measured as functions of stimulation rate, mode, and level. Results show that for all stimulation rate and mode conditions, modulation sensitivity was poorest at quiet listening levels, consistent with results from previous studies. MDTs were better with the lower stimulation rate, especially for quiet-to-medium listening levels. Stimulation mode had no significant effect on MDTs. These results suggest that, although high stimulation rates may better encode temporal information and widen the electrode dynamic range, CI patients may not be able to access these enhanced temporal cues, especially at the lower portions of the dynamic range. Lower stimulation rates may provide better recognition of weak acoustic envelope information.
\end{abstract}

Keywords: modulation detection threshold, stimulation rate, stimulation mode, stimulation level, temporal cues

Correspondence to: John J. Galvin III - Department of Auditory Implants and Perception - House Ear Institute $\bullet 2100$ West Third Street, Los Angeles, CA 90057, USA. email: jgalvin@hei.org

\section{INTRODUCTION}

When spectral information is degraded and/or distorted by cochlear implant (CI) speech processing, temporal envelope cues contribute greatly to speech recognition (Shannon et al. 1995; Turner et al. 1995; van Tasell et al. 1987, 1992). For CI patients and normal-hearing $(\mathrm{NH})$ subjects listening to acoustic CI simulations, slowly varying temporal components $(<20 \mathrm{~Hz})$ provide the most useful phonetic information (Drullman et al. 1994a, b; Fu and Shannon, 2000; Shannon et al. 1995; van Tasell et al. 1987, 1992). Recent studies have also shown that higher-frequency periodicity cues $(50-500 \mathrm{~Hz})$ contribute to perception of suprasegmental information, such as voice gender recognition (Fu et al. 2004) and tone recognition for tonal languages (Fu et al. 1998; Fu and Zeng 2000; Xu et al. 2002).

In CI speech processing, acoustic amplitude envelopes are encoded by modulating the electric current delivered to implanted electrodes. The transmission of acoustic envelope cues is primarily limited by the stimulation rate of the implant device; the small electrode dynamic range (DR) and limited amplitude resolution can further reduce the saliency of temporal cues. Presumably, higher stimulation rates can provide better coding of periodicity cues. Besides providing better temporal sampling, higher stimulation rates may increase the stochastic response properties of the activated neurons (Rubinstein et al. 1999; Wilson et al. 1997a, b), thereby reducing the unnatural phase-locking activity of neural firing patterns. Much recent development of the CI has been toward increasing the stimulation rate to provide better temporal coding, lower stimulation thresholds, and wider dynamic ranges. However, in terms of CI patient performance, no clear advantage 
has been consistently shown with high stimulation rates (Brill et al. 1997, 1998a, b; Friesen et al. 2005; $\mathrm{Fu}$ and Shannon 2000; Holden et al. 2002; Lawson et al. 1996; Loizou et al. 2000; Skinner 2003; Vandali et al. 2000). These studies revealed high intersubject variability; although some patients displayed better recognition with increased stimulation rates, some were not affected by changes in rate and others performed best at one particular rate.

Although stimulation rate may limit the transmission of acoustic envelope cues, CI patients' modulation sensitivity limits the reception of envelope information. Temporal modulation detection has been widely studied in CI and NH listeners (e.g., Bacon and Viemeister 1985; Burns and Viemeister 1981; Donaldson and Viemeister 2000; Formby 1986; Forrest and Green 1987; Fu 2002; Shannon 1992). Significant differences have been noted between CI and NH listeners' modulation sensitivity. For example, CI listeners are generally less sensitive to higher modulation frequencies $(>150 \mathrm{~Hz})$ than $\mathrm{NH}$ listeners. Also, modulation detection thresholds (MDTs) improve strongly with stimulus level in CI listeners, whereas modulation sensitivity is nearly independent of stimulus level for $\mathrm{NH}$ listeners, except at the lowest listening levels. Many studies have also evaluated the relationship between CI patients' modulation sensitivity and speech performance (e.g., Blamey et al. 1992; Cazals et al. 1991, 1994; Fu 2002; Muchnik et al. 1993). Cazals et al. (1994) reported that CI users' speech performance was highly correlated with the cutoff frequency of patients' temporal modulation transfer function (or, TMTF: MDTs as function of modulation frequency). Fu (2002) showed strong individual differences in patients' MDTs as a function of loudness level. Results also showed a highly significant correlation between mean MDTs (averaged over subjects' dynamic range) and phoneme recognition scores. A similar correlation was recently observed in patients with the auditory brainstem implant (Shannon and Colletti 2005).

The inconclusive data from previous studies suggest that, at the very least, not all CI patients may ben- efit from high stimulation rates. Although higher rates may transmit more temporal information, it is unclear whether CI patients can effectively access these cues, especially at lower listening levels where higher-rate envelopes may be coded (e.g., consonants). The present study examined the effects of stimulation rate, mode, and level on CI patients' MDTs for a 20-Hz sinusoidally amplitude modulated pulse train. Relatively high and low stimulation rates were studied to examine the effects of temporal coding and the size of the dynamic range on MDTs. Stimulation mode refers to the electrode configuration used for electrical stimulation. A bipolar stimulation mode refers to stimulation between an active and return intracochlear electrode, whereas a monopolar stimulation mode refers to stimulation between an active intracochlear electrode and an extracochlear return electrode. A narrow stimulation mode (e.g., $\mathrm{BP}+1$ ) refers to a small cochlear distance between the stimulated electrodes, whereas a wide stimulation mode (e.g., BP +13 or monopolar) refers to a large cochlear distance between the stimulated electrodes. Relatively wide and narrow stimulation modes were studied to examine the effects of spread of excitation and the subsequent shift in dynamic range on MDTs. Stimulation levels spanning the entire dynamic range were studied to examine the effects of loudness level on MDTs, across the stimulation rate and mode conditions.

\section{METHODS}

\section{Subjects}

Two Nucleus-24 (N24) and four Nucleus-22 (N22) users participated in the experiment. All CI subjects were postlingually deafened and all had more than two years' experience with their implant device. Relevant subject details are shown in Table 1.

\section{Stimuli}

For all subjects, MDTs were measured with two carrier stimulation rates: 250 pulse per second (pps)

TABLE 1

Relevant information for $\mathrm{Cl}$ users who participated in the experiment

\begin{tabular}{llllcrr}
\hline Subject & Age & Etiology & Prosthesis & $\begin{array}{c}\text { Cl experience } \\
\text { (years) }\end{array}$ & $\begin{array}{c}\text { Vowel recognition } \\
\text { (\% correct) }\end{array}$ & $\begin{array}{c}\text { Consonant recognition } \\
(\% \text { correct) }\end{array}$ \\
\hline S1 & 62 & Hereditary & Nucleus 24 & 2 & 83 & 85 \\
S2 & 72 & Unknown & Nucleus 24 & 5 & 68 & 65 \\
S3 & 45 & Trauma & Nucleus 22 & 13 & 92 & 73 \\
S4 & 61 & Unknown & Nucleus 22 & 15 & 78 & 72 \\
S5 & 61 & Hereditary & Nucleus 22 & 14 & 86 & 75 \\
S6 & 65 & Noise-induced & Nucleus 22 & 9 & 67 \\
\hline
\end{tabular}


and 2000 pps. Although other rates between these experimental rates might also influence MDTs, these rates were chosen because they generally correspond with the rates typically used in speech processors (250 pps for N22 patients fitted with the SPEAK strategy, often nearly 2000 pps for N24 patients fitted with the ACE strategy). All subjects were tested for a relatively narrow electrode configuration $(\mathrm{BP}+3)$. Four subjects were also tested using wider electrode configurations to see whether the spread of excitation would influence modulation sensitivity; monopolar stimulation was tested in two N24 users and BP + 13 stimulation was tested in two N22 users. Table 2 shows the electrode configurations, electrode numbers, and stimulation rates tested for each subject. All stimuli were biphasic pulse trains; the duration was $300 \mathrm{~ms}$, the pulse phase duration was $100 \mu \mathrm{s}$, and the interphase gap was $45 \mu \mathrm{s}$. Stimuli were delivered via custom research interface developed at House Ear Institute (Wygonski and Robert 2002).

For the modulated stimuli, amplitude modulation was applied to the carrier pulse train using the following equation: $[f(t)]\left[1+m \sin \left(2 \pi f_{m} t\right)\right]$, where $f(t)$ is the unmodulated pulse train, $m$ is the modulation index (modulation depth), and $f_{m}$ is the modulation frequency. The modulation depth was adaptively varied during the modulation detection test. The modulation frequency was fixed at $20 \mathrm{~Hz}$.

\section{TABLE 2}

Mean thresholds, mean MALs and estimated DRs for individual subjects and experimental electrodes

\begin{tabular}{lrrrrr}
\hline Subject & Electrode & $\begin{array}{r}\text { Rate } \\
(p p s)\end{array}$ & $\begin{array}{c}\text { Thresh } \\
(d B)\end{array}$ & $\begin{array}{c}M A L \\
(d B)\end{array}$ & $\begin{array}{r}D R \\
(d B)\end{array}$ \\
\hline S1 & $(13,17)$ & 250 & 44.24 & 51.64 & 7.40 \\
& $(13,17)$ & 2000 & 35.74 & 51.40 & 15.67 \\
& $(17)$ & 250 & 36.36 & 46.50 & 10.14 \\
& $(17)$ & 2000 & 28.04 & 46.23 & 18.19 \\
S2 & $(18,22)$ & 250 & 49.44 & 56.68 & 7.24 \\
& $(18,22)$ & 2000 & 42.86 & 55.71 & 12.85 \\
& $(22)$ & 250 & 38.37 & 45.74 & 7.37 \\
& $(22)$ & 2000 & 30.74 & 45.04 & 14.30 \\
S3 & $(14,18)$ & 250 & 48.51 & 59.10 & 10.59 \\
& $(14,18)$ & 2000 & 40.43 & 58.95 & 18.52 \\
& $(4,18)$ & 250 & 39.80 & 50.98 & 11.18 \\
& $(4,18)$ & 2000 & 33.15 & 49.09 & 15.95 \\
S4 & $(14,18)$ & 250 & 48.82 & 54.19 & 5.37 \\
& $(14,18)$ & 2000 & 41.99 & 53.29 & 11.31 \\
& $(4,18)$ & 250 & 42.43 & 48.60 & 6.17 \\
& $(4,18)$ & 2000 & 35.17 & 45.21 & 10.05 \\
S5 & $(14,18)$ & 250 & 50.44 & 55.78 & 5.34 \\
& $(14,18)$ & 2000 & 42.32 & 54.36 & 12.05 \\
S6 & $(14,18)$ & 250 & 46.82 & 53.46 & 6.64 \\
& $(14,18)$ & 2000 & 38.94 & 51.30 & 12.36 \\
\hline
\end{tabular}

Threshold and MAL values are in $\mathrm{dB}$ re: $1 \mu \mathrm{A}$. DR was calculated as the difference between MAL and threshold in $\mathrm{dB}$.
Dynamic range estimation and loudness balancing

Before beginning the modulation detection experiment, the dynamic range (DR) was estimated for all experimental electrodes. Absolute detection thresholds for unmodulated pulse trains were measured using an adaptive three-alternative forced-choice (3AFC) procedure (3-down/1-up). During the test, the stimulus was randomly presented to one of the three intervals. The amplitude of the stimulus was adjusted according to subject response. The final 8 of 12 reversals for each run were averaged to obtain the threshold. Three to six test runs were conducted for each electrode pair, and the means from all test runs were averaged to obtain the mean threshold for each electrode pair. Maximum acceptable loudness (MAL) levels were obtained by using a method of limits; MAL was defined as the loudest sound that the subject would be willing to listen to for an extended period of time (e.g., during a psychophysical test). The experimenter slowly and incrementally raised the stimulation level until the subject reported that MAL was achieved. Three to six test runs were conducted for each electrode pair, and the means from all test runs were averaged to obtain the mean MAL. For each electrode pair, the estimated DR was obtained by subtracting the mean threshold from the mean MAL. Mean threshold, mean MAL, and estimated DR are shown for individual subjects and experimental electrodes in Table 2. Threshold and MAL levels are reported in $\mathrm{dB}$ re: $1 \mu \mathrm{A}$.

To equate loudness levels across experimental conditions, presentation levels were loudness-balanced to reference levels of a reference electrode pair. Note that only unmodulated pulse trains were loudness-balanced. For all subjects (except S2), electrode pair $(9,13)$ served as the reference electrode pair. Electrode pair $(14,18)$ served as the reference electrode pair for S2 because of the limited electrode insertion depth. For the reference electrode pair, the stimulation rate was $1000 \mathrm{pps}$, the duration was 300 $\mathrm{ms}$, the pulse phase duration was $100 \mu \mathrm{s}$, and the interphase gap was $45 \mu \mathrm{s}$. The DR for the reference electrode pair was estimated using the methods described above; six threshold and six MAL measurements were used to estimate the DR. Eight reference levels were linearly distributed across the DR (in $\mu \mathrm{A})$, corresponding to $5 \%, 15 \%, 25 \%, 35 \%, 45 \%, 55 \%$, $65 \%$, and $75 \%$ of the reference electrode pair's DR. Stimulation levels for the experimental stimulation rate and mode conditions were loudness-balanced to these reference levels using a 2AFC, double-staircase procedure (Jesteadt 1980; Zeng and Turner 1991). For each reference level, the amplitude of the experimental electrode was adjusted according to subject 
response. For each experimental electrode pair, the means of four to six loudness-balanced amplitudes for each reference level were used as presentation levels for the modulation detection experiments. Thus, across the stimulation rate and mode conditions, stimulation levels were loudness-balanced for listening levels ranging from very soft to comfortably loud.

\section{Modulation detection}

MDTs were measured for each stimulation rate and mode condition as a function of loudness level. An adaptive 3AFC procedure was used in which the modulation depth was varied according to subject response (3-down/1-up). Two intervals (randomly selected) contained unmodulated pulse trains, whereas the third interval (randomly selected) contained the modulated pulse train. The test electrode, stimulation rate, and stimulation level were fixed during each test run; only the modulation depth was varied from trial to trial. The final 8 of 12 reversals for each run were averaged to obtain the MDT; three to six test runs were conducted for each experimental electrode pair at each listening level. The modulation thresholds (in percent) were converted to $\log$ scale $(20 \log$ $m$ ) to allow for easier comparison across test conditions and listening levels.

\section{RESULTS}

Table 2 shows the thresholds, MALs, and DRs for each experimental electrode. For all subjects, the DR was significantly larger with the 2000 pps stimulation rate than with the 250 pps rate (paired Student's $t$ test, $p<0.001)$. The increased DR with the high rate was largely a result of lower detection thresholds; threshold levels were significantly lower with the high rate $(p<0.001)$; MALs were slightly but significantly lower with the higher rate $(p=0.005)$. For N24 users S1 and S2, monopolar stimulation also resulted in slightly, but not significantly larger DRs, relative to bipolar stimulation, for both stimulation rates $(p=$ $0.065)$; threshold $(p=0.003)$ and MAL $(p=0.016)$ levels were significantly lower with monopolar stimulation. For N22 users S3 and S4, stimulation mode did not significantly affect the size of the DR $(p=$ $0.501)$; threshold $(p<0.001)$ and MAL $(p=0.003)$ levels were significantly lower with $\mathrm{BP}+13$ stimulation, effectively shifting the DR to lower current levels. In general, stimulation rate seemed to have the greatest effect on DRs, primarily because of the lower detection thresholds with the 2000 pps rate. Changes in stimulation mode largely resulted in shifted DRs, with small increases in DRs sometimes associated with monopolar or wide bipolar electrode configurations.

Figure 1 shows individual CI subjects' modulation detection functions for high and low carrier stimulation rates, as a function of listening level, for a fixed stimulation mode $(\mathrm{BP}+3)$. Each panel shows MDTs for an individual subject with the high and low carrier rates. The $x$-axis shows the reference loudness level (in percent DR of the reference electrode used in the loudness-balancing procedure); thus, at each reference level, the MDTs for the different carrier rates were for equally loud listening levels. The $y$-axis shows the MDT in $\log$ scale $(20 \log m)$; note that lower values represent better modulation sensitivity. The filled circles represent MDTs with the 2000 pps carrier and the open circles represent MDTs with the 250 pps carrier. The dashed and solid lines show sigmoidal fits to the MDT data for the 250 and 2000 pps carriers, respectively.

There were significant differences in the MDT functions across subjects and stimulation rates. For all subjects, MDTs were most significantly affected by the loudness level. In general, MDTs improved as the loudness level was increased, for both stimulation rates. Also, for most subjects, MDTs were better with the 250 pps carrier than with the 2000 pps carrier, especially at the lower presentation levels. A two-way repeated measures ANOVA showed that MDTs were significantly affected by the stimulation rate $\left(F_{1,35}=\right.$ $30.3, p=0.003)$ and level $\left(F_{7,35}=35.3, p<0.001\right)$. There was a significant interaction between stimulation rate and level $\left(F_{7,35}=30.6, p=0.019\right)$, reflected by the better MDTs with the 250 pps carrier at the lower listening levels.

Figure 2 shows the difference in performance between the two carrier rates for the $\mathrm{BP}+3$ stimulation mode. The $x$-axis shows the reference loudness level (as in Fig. 1). The $y$-axis shows the difference in MDTs between the two carrier rates, in dB. For each subject, at each reference level, the MDT with the 2000 pps carrier was subtracted from the MDT with the 250 pps carrier; negative values indicate better MDTs with the $250 \mathrm{pps}$ carrier. Individual subject data are shown by the different symbols. Filled symbols indicate a significant difference in MDTs between the carrier rates $(t$ test, $p<0.05)$, whereas open symbols indicate no significant difference in MDTs between the carrier rates $(t$ test, $p>0.05)$. The solid line represents the mean shift in MDTs between the carrier rates, across subjects. As seen in the figure, for most subjects, MDTs were significantly better with the 250 pps carrier, especially at the lower presentation levels. A similar pattern was observed with the monopolar and BP +13 stimulation modes.

For widely spaced electrode configurations (monopolar or $\mathrm{BP}+13)$, stimulation rate and level had a 

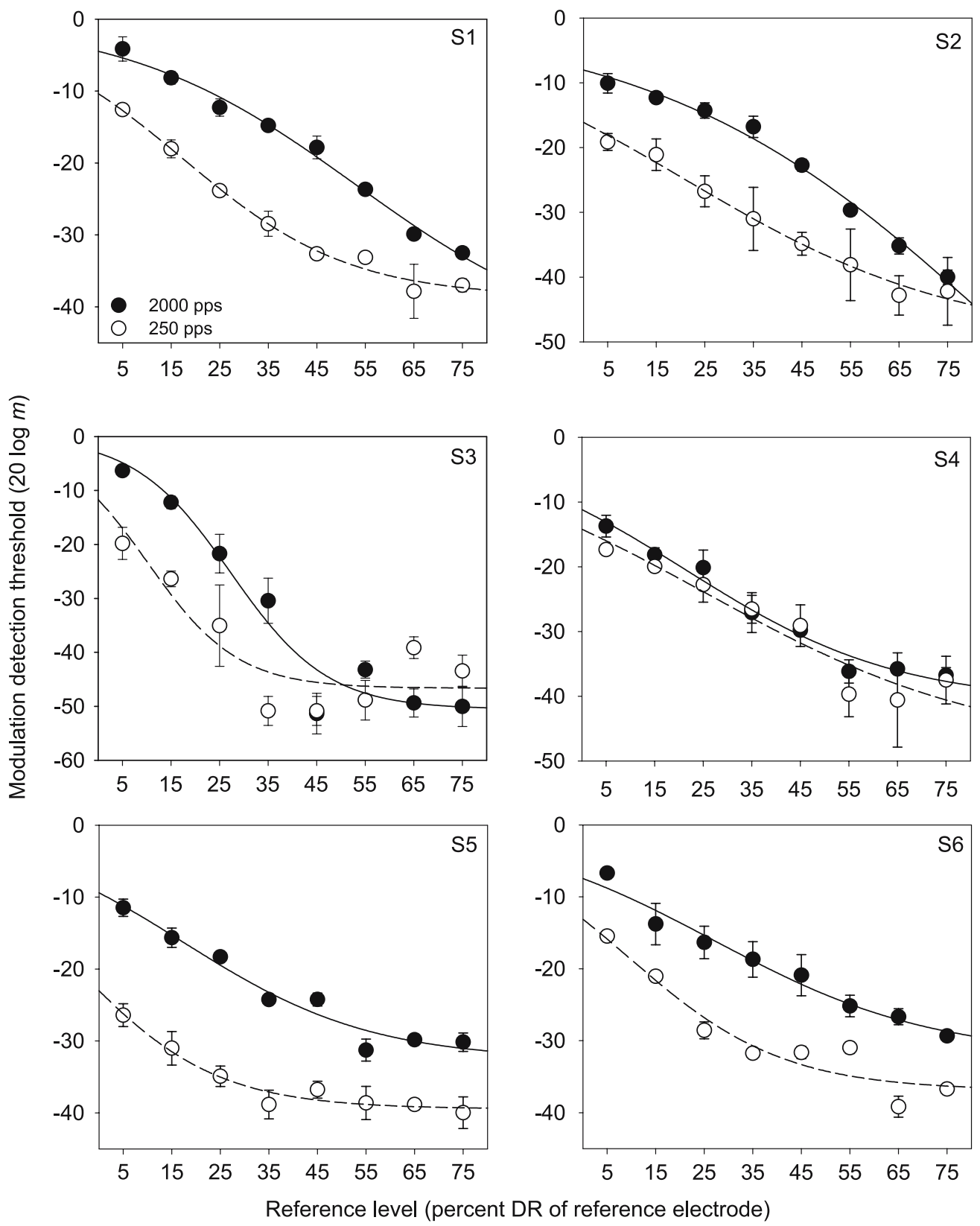

FIG. 1. Modulation detection thresholds (MDTs) for individual $\mathrm{Cl}$ subjects. Each panel shows individual subject results. The $x$-axis shows the reference loudness level, in percent DR of the reference electrode. The $y$-axis shows the MDTs in log scale; note that the scale is optimized for each subject's range of MDTs. The filled cir-

similar effect on CI users' modulation sensitivity. A two-way repeated-measures ANOVA showed that MDTs were significantly affected by the stimulation rate $\left(F_{1,21}=13.7, p=0.034\right)$ and level $\left(F_{7,21}=38.9, p\right.$ $<0.001)$. There was a significant interaction between stimulation rate and level $\left(F_{7,21}=4.27, p=0.004\right)$. Thus, the effects of stimulation rate and level were similar for both relatively narrow $(\mathrm{BP}+3)$ and broad (monopolar, BP +13$)$ stimulation modes. Figure 3 cles represent MDTs with the 2000 pps carrier and the open circles represent MDTs with the 250 pps carrier; the error bars represent 1 standard deviation. The solid and dashed lines represent sigmoid fits to the MDT data for the 2000 and 250 pps carriers, respectively.

shows the shift in MDTs (in $\mathrm{dB}$ ) between the two stimulation mode conditions, for a fixed carrier rate (250 pps). Similar to Figure 2, the $x$-axis shows the reference loudness level; the $y$-axis shows the difference in MDTs between the two stimulation mode conditions, in $\mathrm{dB}$. For each subject, the MDT with $\mathrm{BP}+3$ stimulation was subtracted from the MDT with either monopolar or BP + 13 stimulation (depending on the subject) at each reference level. 


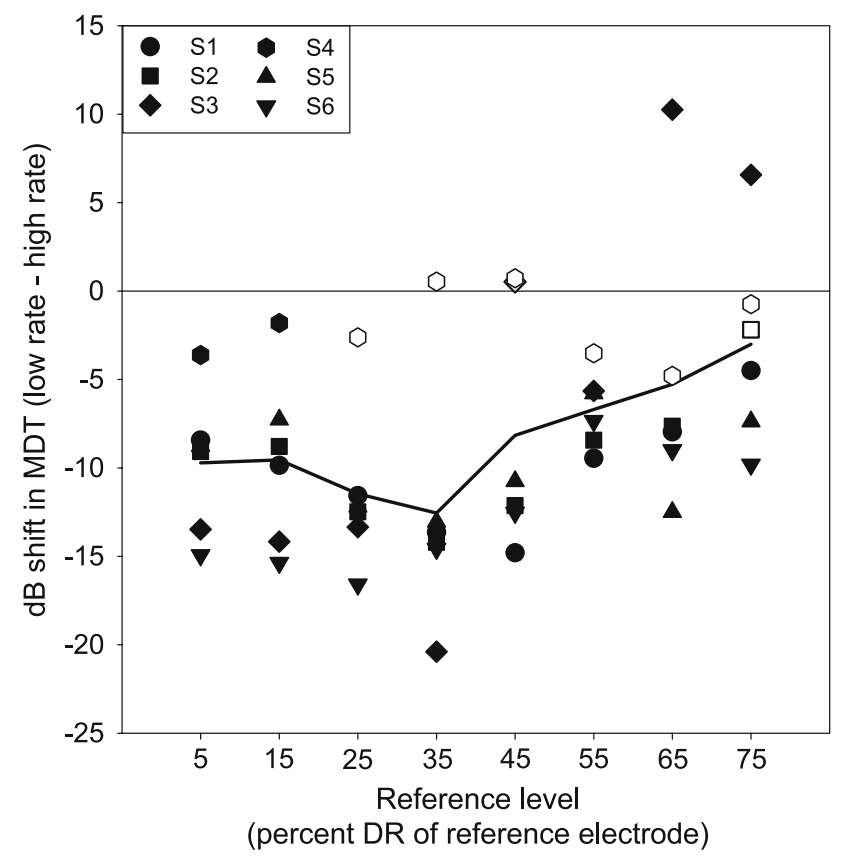

FIG. 2. Shift in MDTs between stimulation rates, for BP +3 stimulation. For each subject, at each loudness-balanced reference level, the MDT with the 2000 pps carrier was subtracted from the MDT with the 250 pps carrier. The $x$-axis shows the reference loudness level, in percent dynamic range of the reference electrode. The $y$-axis shows the difference in MDTs between the carrier rates (in $\mathrm{dB}$ ). The reference line at $0 \mathrm{~dB}$ represents no difference in MDTs between the carrier rates. The thick solid line shows the mean performance shift, across subjects. Individual subject data are represented by the different symbols. The filled symbols represent a statistically significant difference in MDTs between the carrier rates (paired $t$ test: $p<0.05$ ); the open symbols represent differences in MDTs that were not statistically significant between the carrier rates $(p>0.05)$.

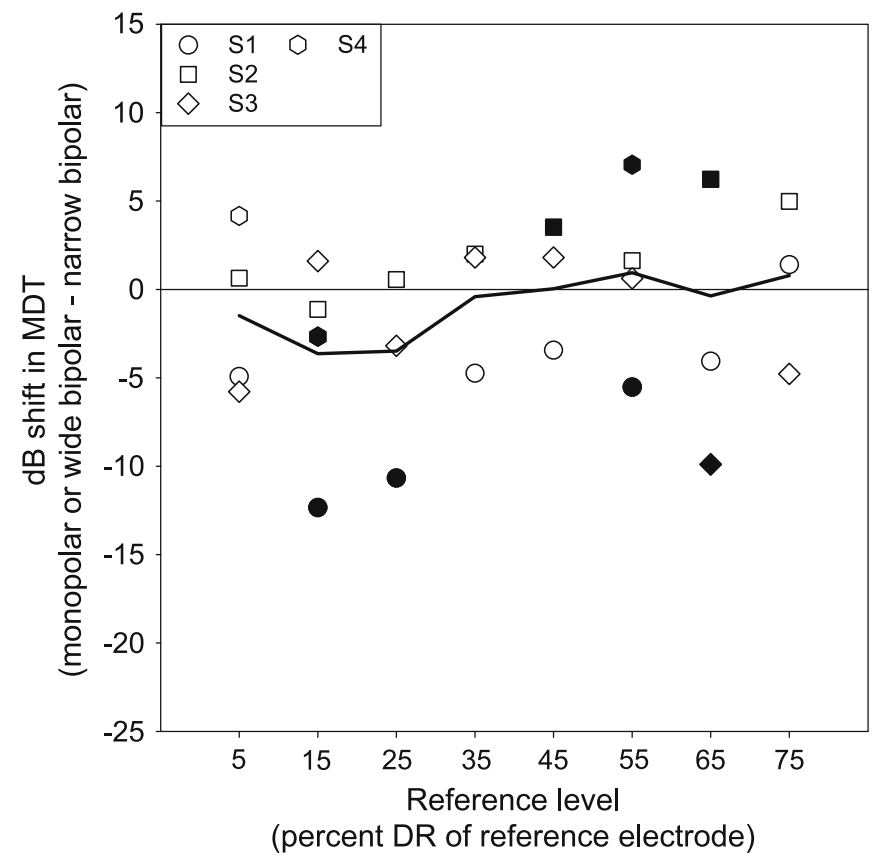

FIG. 3. Shift in MDTs between stimulation modes, for 250 pps stimulation rate. For subjects S1 and S2, the MDT with the BP + 3 configuration was subtracted from the MDT with the monpolar configuration. For subjects S3 and S4, the MDT with the BP +3 configuration was subtracted from the MDT with the BP +13 configuration. The $x$-axis shows the reference loudness level, in percent dynamic range of the reference electrode. The $y$-axis shows the difference in MDTs between the electrode configurations (in $\mathrm{dB}$ ).
The reference line at $0 \mathrm{~dB}$ represents no difference in MDTs between the carrier rates. The thick solid line shows the mean performance shift, across subjects. Individual subject data are represented by the different symbols. The filled symbols represent a statistically significant difference in MDTs between the carrier rates $(t$ test: $p<$ $0.05)$; the open symbols represent differences in MDTs that were not statistically significant between the carrier rates $(p>0.05)$. 


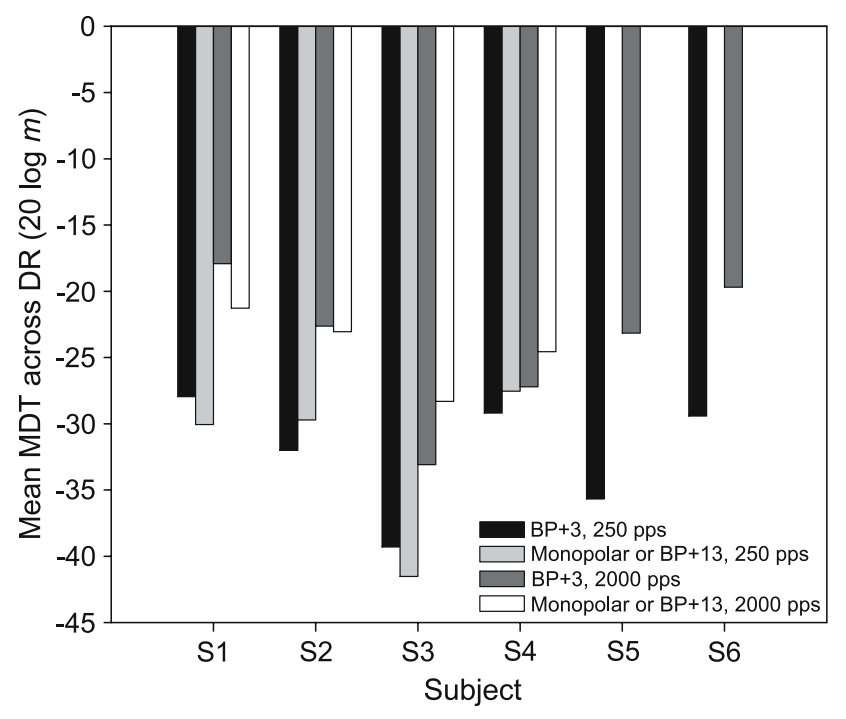

FIG. 4. Mean MDTs (across entire DR). The $x$-axis shows individual subjects. The $y$-axis shows the mean MDT in dB; for each subject and condition, MDTs were averaged across all reference levels. The bars represent different electrode configuration and carrier rate conditions.

Individual subject data are shown by the different symbols. Filled symbols indicate a significant difference in MDTs between the carrier rates ( $t$ test, $p<0.05)$, whereas open symbols indicate no significant difference in MDTs between the carrier rates $(p>0.05)$. The solid line represents the mean shift in MDTs between the stimulation modes, across subjects.

For most subjects, there was no significant difference in MDTs between the stimulation mode conditions at most presentation levels. For the 250 pps stimulation rate, a two-way repeated-measures ANOVA showed that MDTs were significantly affected by stimulation level $\left(F_{7,21}=28.8, p<0.001\right)$; however, stimulation mode had no effect $\left(F_{1,21}=0.006, p=\right.$
$0.94)$ and there was no significant interaction between stimulation level and mode $\left(F_{7,21}=0.57, p=\right.$ 0.77). Similarly, for the 2000 pps stimulation rate, a two-way repeated measures ANOVA showed that MDTs were significantly affected by stimulation level $\left(F_{7,21}=26.9, p<0.001\right)$; however, stimulation mode had no effect $\left(F_{7,21}=0.263, p=0.64\right)$ and there was no significant interaction between stimulation level and mode $\left(F_{7,21}=0.32, p=0.935\right)$.

To find more global effects of stimulation mode and rate on CI subjects' modulation sensitivity, mean MDTs were calculated across all reference levels for each subject, for each configuration/rate condition. Figure 4 shows mean MDTs (across all stimulation levels) for individual subjects. The differently

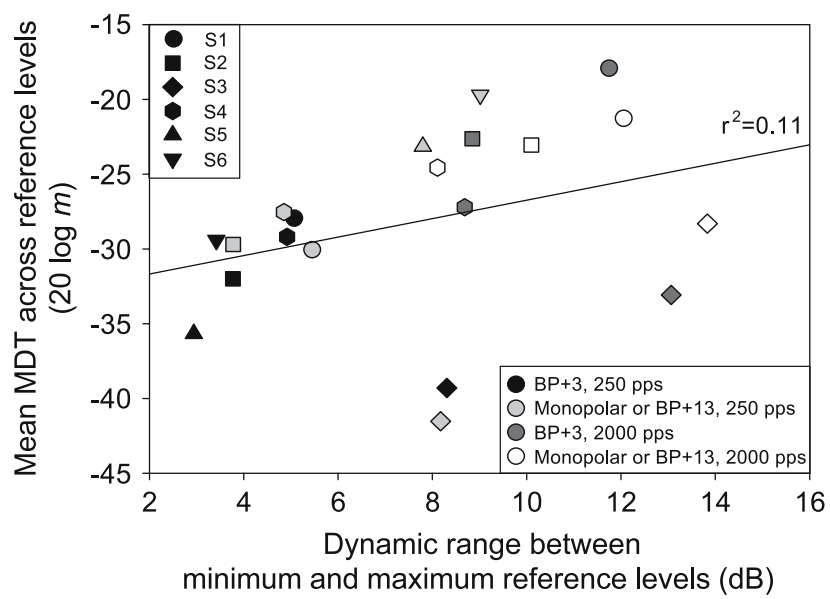

FIG. 5. Mean MDTs (across the range of reference levels) as a function of dynamic range (measured between the minimum and maximum reference levels). Individual subject data are represented by the different symbols; the different stimulation mode/rate conditions are represented by the different fill shades. The solid line represents the linear regression for all data. 
shaded bars represent the different stimulation rate/ mode conditions. For the four subjects who completed all stimulation rate/mode conditions, a twoway repeated measures ANOVA showed that mean MDTs were significantly affected by stimulation rate $\left(F_{1,3}=19.4, p=0.022\right)$; again, stimulation mode had no effect $\left(F_{1,3}=0.144, p=0.730\right)$ and there was no significant interaction between stimulation rate and mode $\left(F_{1,3}=0.221, p=0.670\right)$. Although individual subjects may have differed in terms of mean modulation sensitivity across the DR, mean modulation sensitivity was consistently better with the lower stimulation rate.

Figure 5 shows subjects' mean MDTs (calculated across all reference levels, as in Figure 4) as a function of the DR (between the minimum and maximum loudness-balanced reference levels). Individual subject data are shown by different symbols and the different test conditions are shown by the fill shades. A nonsignificant linear regression across all subject data showed no correlation between the DR and modulation sensitivity $\left(r^{2}=0.11\right)$.

\section{DISCUSSION}

Results from the present study demonstrated that carrier stimulation rate strongly affected CI subjects' modulation sensitivity, especially at softer listening levels. This result is somewhat contradictory to the findings of Wojtczak and Viemeister (1999), who found that MDTs were similar for sinusoidal carriers with rates between 250 and $8000 \mathrm{~Hz}$ in NH listeners. Differences in experimental design and subject population between the present study and the Wojtczak and Viemeister study may account for several differences in results. In $\mathrm{NH}$ listeners, the location and rate of neural firing cannot be easily isolated. Thus, in the Wojtczak and Viemeister study, the 250 and $8000 \mathrm{~Hz}$ carriers would have stimulated different tonotopic locations; stimulation at either rate would have produced stochastic, primary neural response patterns at each of these locations. In CI patients, the effect of stimulation rate can be evaluated independent of the tonotopic location. Thus, in the present study, the two experimental carrier stimulation rates were evaluated for the same electrode location, allowing the temporal processing effects between the two rates to be compared for (presumably) the same neural population. Differences between the stimulation levels used in the two studies may have also contributed to differences in results; MDTs were most comparable between the carrier rates at the louder listening levels and most different at the softer levels. The stimulation rates used in the present study also produced very different DRs, which may have contributed to differences in MDTs at different loudness levels. Finally, different modulation frequencies were evaluated (4 $\mathrm{Hz}$ in the Wojtczak and Viemeister study, $20 \mathrm{~Hz}$ in the present study), which may have involved different degrees of temporal processing between the two studies.

The strong effect of stimulation rate on MDTs may be a result of the limits of temporal processing or because of the large differences in DR between the carrier rates. Neural firing has been shown to synchronize with rate cycles of up to $\sim 1000 \mathrm{~Hz}$ in human electric hearing (Wilson et al. 1997a, b). At higher stimulation rates, low levels of neural noise due to refractoriness and/or discharge of neural membranes may introduce a jitter to the neural response patterns. Higher stimulation rates and/or conditioning pulse trains have been employed in contemporary speech processors with the aim of desynchronizing neural firing patterns and thereby restoring some of the spontaneous firing patterns observed in NH listeners (Wilson et al. 1997a, b; Rubinstein et al. 1999, Litvak et al. 2001). Although discharge rate patterns in auditory nerves may indicate some improvement in temporal coding in animals and models, such improved coding has not been explicitly and/or consistently shown to improve speech recognition in CI users. In studying the cortical physiology of the guinea pig, Middlebrooks (2004) measured the effects of stimulation rate on channel interaction and theorized that high rates might induce temporal integration due to depolarization of the cochlear neural membrane, which would contribute in part to the steeply declining thresholds observed with rates above 1000 pps. Such a depolarization might also influence temporal processing on a single electrode, in which temporal smearing, due to depolarization/polarization time constants, might weaken the salience of the amplitude envelope. Recently, Middlebrooks (2005) reported that MDTs in guinea pigs were poorer at higher carrier rates; in that study, MDTs were measured for incremental stimulation levels above threshold. It is possible that the relative loudness between the carrier rates was not comparable and that the differences in MDTs were a result of the differences in loudness. However, in the present study, MDTs were compared across carrier rates at loudness-balanced listening levels, producing similar results to Middlebrooks' recent findings.

Changes in stimulation mode did not strongly affect CI subjects' modulation sensitivity. Although the spread of excitation will certainly contribute to loudness and, to some extent, place-pitch per- 
ception, loudness seems to contribute most strongly to many psychophysical measures (e.g., intensity discrimination, modulation detection, stimulation rate discrimination, etc.). The effect of stimulation mode on MDTs was evaluated in the present experiment to test whether representation of the envelope would be enhanced by a larger spatial spread of excitation or by the change/shift in DR. Chatterjee (2003) showed that channel interaction increased when synchronized envelopes were presented to tonotopically remote electrodes. Modulation detection interference also increases when modulated maskers are presented to tonotopically remote electrode locations (Chatterjee and Oba 2005). In some way, these data imply that amplitude envelope cues might be more salient when presented to a wide region of the cochlea (e.g., BP + 13 and monopolar stimulation modes used in the present study) rather than a restricted region (e.g., $\mathrm{BP}+3$ in the present study). Morris and Pfingst (2000) found that, when stimulation levels were equated for loudness, stimulation mode did not affect stimulation rate discrimination. Similarly, Franck et al. (2003) found that speech recognition was not affected by stimulation mode, but rather by stimulation level, and that the spread of excitation (bipolar vs. monopolar stimulation) did not seem to influence CI speech performance. Thus, it is not surprising that stimulation mode, while influencing the spread of excitation and thereby shifting the electrode DR, did not affect CI subjects' MDTs in the present study.

Differences in stimulation level most strongly affected CI subjects' modulation sensitivity. Although the stimulation mode did not affect MDTs and stimulation rate contributed to a 20-dB difference (at most) in MDTs, the difference in MDTs between the loudest and softest stimulation levels was between 15 and $40 \mathrm{~dB}$, across CI subjects. A significant interaction was found between stimulation rate and level, but not between stimulation level and mode. Note that the DR was significantly larger between the stimulation rate conditions (and not between stimulation mode conditions). This interaction suggests that MDTs were affected differently by stimulation rate at different loudness levels. The interaction may have been a result of differences in loudness growth between the two rates and/or differences between intensity difference limens (DLs) between rates at equally loud presentation levels. The strong level dependence of modulation sensitivity requires that other processor parameters (e.g., stimulation rate, stimulation mode, modulation frequency, etc.) must be evaluated for a wide range of listening levels, as in the present study, to best ascertain their effects.
Because the stimulation rate most significantly affected the DRs of each carrier rate, the DR might be expected to be a limiting factor in CI patients' modulation sensitivity. However, Figure 5 shows no correlation between mean MDTs (averaged across the reference listening levels) and DRs (measure between the minimum and maximum reference levels). The distribution of data shows a trend in which the smaller DRs (due to the lower carrier rates) produced lower MDTs. Although subject S3's data (diamond symbols) agrees with this trend, S3's DRs were larger and MDTs lower than those of most other subjects. It should be noted that S3 was implanted for hearing loss resulting from trauma; all other subjects were postlingually deafened with progressive hearing losses. When S3's data are excluded, the significance of the correlation greatly increases (from $r^{2}=0.11$ to $\left.r^{2}=0.81\right)$. The data suggest that gains in DR as a result of higher stimulation rates are offset by increased MDTs. A similar observation was found in the relation between DR and intensity DLs (Kreft et al. 2004). One implication from these results is that gains in DR do not provide better amplitude resolution and therefore do not provide for better envelope processing.

The presumed advantages for high-rate stimulation seem to be offset by increased channel interaction (Middlebrooks 2004), poorer intensity resolution (Kreft et al. 2004), and the poorer MDTs shown in the present study. Although high stimulation rates offer more flexibility in CI speech processing schemes, improved temporal sampling and increased DRs may not be the most effective use of the technology. If more spectral channels may be added (whether by increasing the number of implanted electrodes or by improving the "effective" number of spectral channels by other means), the increased cumulative rate might be better distributed among these additional channels, rather than increasing the single-channel stimulation rate.

The strong carrier rate effects and level dependence of modulation sensitivity suggest that weak envelope cues are most affected by speech processor parameters. Much speech perception, especially for clear speech under quiet listening conditions, may not require the full extent of CI patients' modulation sensitivity, especially if there is adequate spectral resolution. However, for speech recognition tasks requiring the processing of low-amplitude envelopes (e.g., consonant recognition, speech in noise, etc.), modulation sensitivity may play a greater role. The mixed results of high rate processors on CI users' speech recognition may be a result of variability in various performance measures in these studies. If high-rate speech processors are thought to encode and transmit more temporal information, they must 
be tested using tasks that are sensitive to temporal cues. Simple sentence recognition tests under quiet listening conditions may not be sensitive to these temporal cues. Thus, future work comparing the effects of stimulation rate on CI patient performance must include speech tests that are sensitive to the temporal cues provided by each rate condition.

\section{SUMMARY AND CONCLUSION}

Modulation detection thresholds (MDTs) for a $20-\mathrm{Hz}$ sinusoidally amplitude modulated pulse train were measured in six cochlear implant users, as functions of the stimulation rate, mode, and level. Major findings include:

1. MDTs were sensitive to stimulation rate, especially at lower stimulation levels. For equally loud stimulation levels, MDTs were generally better with the 250 pps carrier than with the 2000 pps carrier.

2. MDTs were not sensitive to the stimulation mode. For equally loud stimulation levels, there was no significant difference between $\mathrm{BP}+3, \mathrm{BP}+13$, and monopolar stimulation modes.

3. MDTs were most sensitive to stimulation level. In most implant subjects, modulation thresholds worsened as the stimulation level was decreased, consistent with previous studies.

4. Modulation sensitivity seems to be related to intensity resolution (which in turn is related to the dynamic range). Because intensity resolution is fairly constant across manipulations of factors that influence dynamic range (e.g., stimulation mode, rate) within a given CI patient, it is not surprising that MDTs were no better with the high stimulation rate (which produced a much wider dynamic range than with the lower stimulation rate). The fact that MDTs were significantly worse with high stimulation rates may be because of other temporal processing deficits associated with high stimulation rates.

5. Although high stimulation rates may provide better temporal sampling of the acoustic envelope, CI listeners may be unable to access these additional temporal cues and may ultimately receive less temporal information than that provided by lower stimulation rates.

\section{ACKNOWLEDGMENTS}

The authors would like to thank all the CI patients who graciously participated in these experiments. We would also like to thank Dr. Monita Chatterjee for general guidance and Dr. Chris Turner and another anonymous reviewer for their insightful comments. This work was supported by NIDCD R01-004993.

\section{REFERENCES}

BACON SP, VIEMEISTER NF. Temporal modulation transfer functions in normal-hearing and hearing-impaired listeners. Audiology 24:117-134, 1985.

Blamey PJ, Pyman BC, Gordon M, Clark GM, Brown AM, Dowell RC, Hollow RD. Factors predicting postoperative sentence scores in postlinguistically deaf adult cochlear implant patients. Ann. Otol. Rhinol. Laryngol. 101:342-348, 1992.

Brill SM, Gstöttner W, Helms J, Ilberg CV, Baumgartner W, MÜller J, Kiefer J. Optimization of channel number and stimulation rate for the fast continuous interleaved sampling strategy in the COMBI 40+. Am. J. Otol. 18:S104S106, 1997.

Brill SM, Hochmair I, Hochmair ES. The importance of stimulation rate in pulsatile stimulation strategies in cochlear implants. Presented at the XXIV International Congress of Audiology, Buenos Aires, 1998a.

Brill SM, Schatzer R, Nopp P, Hochmair I, Hochmair ES. JCIS: CIS with temporally jittering stimulation pulses: effect of jittering amplitude and stimulation rate on speech understanding. Presented at the 4th European Symposium on Paediatric Cochlear Implantation, Hertogenbosch, The Netherlands, 1998b.

Burns EM, VIEMeISTER NF. Played-again SAM: further observations on the pitch of amplitude-modulated noise. J. Acoust. Soc. Am. 70:1655-1660, 1981.

Cazals Y, Pelizzone M, Kasper A, Montandon P. Indication of a relation between speech perception and temporal resolution for cochlear implantees. Ann. Otol. Rhinol. Laryngol. 100:893895, 1991.

Cazals Y, Pelizzone M, Saudan O, Boex C. Low-pass filtering in amplitude modulation detection associated with vowel and consonant identification in subjects with cochlear implants. J. Acoust. Soc. Am. 96:2048-2054, 1994.

Chatterjee M. Modulation masking in cochlear implant listeners: envelope versus non seq. tonotopic components. J. Acoust. Soc. Am. 113:2042-2053, 2003.

Chatterjee M, Ова SI. Across- and within-channel envelope interactions in cochlear implant listeners. J. Assoc. Res. Otolaryngol. 5:360-375, 2005.

Donaldoson GS, Viemeister NF. Intensity discrimination and detection of amplitude modulation in electric hearing. J. Acoust. Soc. Am. 108:760-763, 2000.

Drullman R, Festern JM, Plomp R. Effect of temporal envelope smearing on speech reception. J. Acoust. Soc. Am. 95:10531064, 1994a.

Drullman R, Festern JM, Plomp R. Effect of reducing slow temporal modulations on speech reception. J. Acoust. Soc. Am. 95: 2670-2680, 1994b.

Formby C. Modulation detection by patients with eighth-nerve tumors. J. Speech Hear. Res. 29:413-419, 1986.

Forrest TG, GREen DM. Detection of partially filled gaps in noise and the temporal modulation transfer function. J. Acoust. Soc. Am. 82:1933-1943, 1987.

Franck KH, Xu L, Pfingst BE. Effects of stimulus level on speech perception with cochlear prostheses. J. Assoc. Res. Otolaryngol. 4:49-59, 2003.

Friesen LM, Shannon RV, Cruz RJ. Effects of stimulation rate on speech recognition with cochlear implants. Audiol. Neuro-otol. 10:169-184, 2005. 
Fu QJ. Temporal processing and speech recognition in cochlear implant users. NeuroReport 13:1635-1640, 2002.

Fu QJ, Shannon RV. Effects of stimulation rate on phoneme recognition in cochlear implant users. J. Acoust. Soc. Am. 107:589-597, 2000.

Fu QJ, Zeng FG. Effects of envelope cues on Mandarin Chinese tone recognition. Asia-Pac. J. Speech Lang. Hear. 5:45-57, 2000.

Fu QJ, Zeng FG, Shannon RV, Soli SD. Importance of tonal envelope cues in Chinese speech recognition. J. Acoust. Soc. Am. 104:505-510, 1998.

Fu QJ, Chinchilla S, Galvin JJ. The role of spectral and temporal cues in voice gender discrimination by normal-hearing listeners and cochlear implant users. J. Assoc. Res. Otolaryngol. 254-260, 2004.

Holden LK, Skinner MW, Holden TA, Demorest ME. Effects of stimulation rate with the Nucleus 24 ACE speech coding strategy. Ear Hear. 23:463-476, 2002.

JesteAdT W. An adaptive procedure for subjective judgments. Percept. Psychophys. 28:85-88, 1980.

Kreft HA, Donaldson GS, Nelson DA. Effects of pulse rate and electrode array design on intensity discrimination in cochlear implant users. J. Acoust. Soc. Am. 116:2258-2268, 2004.

Lawson DT, WiLson BS, Zerbi M, Finley CC. Speech processors for auditory prostheses. Third Quarterly Progress Report, NIH Contract N01-DC-5-2103, 1996.

Litvak L, Delgutte B, Eddington D. Auditory nerve fiber responses to electric stimulation: modulated and unmodulated pulse trains. J. Acoust. Soc. Am. 110:368-379, 2001.

Loizou PC, Poroy O, Dorman MF. The effect of parametric variations of cochlear implant processors on speech understanding. J. Acoust. Soc. Am. 108:790-802, 2000.

Middlebrooks JC. Effects of cochlear-implant pulse rate and interchannel timing on channel interactions and thresholds. J. Acoust. Soc. Am. 116:452-468, 2004.

Middlebrooks JC. Transmission of temporal information from a cochlear implant to the auditory cortex. Abstracts of Association for Research in Otolaryngology 28th Midwinter Meeting, February 2005, Volume 28, 91, 2005.

Morris DJ, Pfingst BE. Effects of electrode configuration and stimulus level on rate and level discrimination with cochlear implants. J. Assoc. Res. Otolaryngol. 1:211-223, 2000.

Muchnik C, Taitelbaum R, Tene S, Hildesheimer M. Auditory temporal resolution and open speech recognition in cochlear implant recipients. Scand. Audiol. 23:105-109, 1993.
Rubinstein JT, Wilson BS, Finley CC, Abbas PJ. Pseudospontaneous activity: stochastic independence of auditory nerve fibers with electrical stimulation. Hear. Res. 127:108-118, 1999.

SHANNON RV. Temporal modulation transfer functions in patients with cochlear implants. J. Acoust. Soc. Am. 91:2156-2164, 1992.

Shannon RV, Colletti V. Evidence from auditory brainstem implants of a modulation-specific auditory pathway that is critical for speech recognition. Abstracts of Association for Research in Otolaryngology 28th Midwinter Meeting, February 2005, Volume 28, 183, 2005.

Shannon RV, Zeng FG, Wygonski J, Kamath V, Ekelid M. Speech recognition with primarily temporal cues. Science 270:303-304, 1995.

SkINNER MW. Optimizing cochlear implant speech performance. Ann. Otol. Rhinol. Laryngol. Suppl. 191:4-13, 2003.

Turner CW, Souza PE, Forget LN. Use of temporal envelope cues in speech recognition by normal and hearing-impaired listeners. J. Acoust. Soc. Am. 97:2568-2576, 1995.

van Tasell DJ, Soli SD, Kirby VM, Widin GP. Speech waveform envelope cues for consonant recognition. J. Acoust. Soc. Am. 82:1152-1161, 1987.

van Tasell DJ, Greenfield DG, Logemann JJ, Nelson DA. Temporal cues for consonant recognition: training, talker generalization, and use in evaluation of cochlear implants. J. Acoust. Soc. Am. 92:1247-1257, 1992.

Vandali AE, Whitford LA, Plant KL, Clark GM. Speech perception as a function of electrical stimulation rate: using the Nucleus 24 cochlear implant system. Ear Hear. 21:608-624, 2000.

Wilson BS, Finley CC, Lawson D, Zerbi M. Temporal representations with cochlear implants. Am. J. Otol. 18:S30-S34, 1997a.

Wilson B, Finley C, Zerbi M, Lawson D, van den Honert C. Speech processors for auditory prostheses. NIH Project N01-DC-5-2103, Seventh Quarterly Progress Report, Neural Prosthesis Program, National Institutes of Health, Bethesda, MD, 1997b.

WojtcZaK M, ViemeIster NF. Intensity discrimination and detection of amplitude modulation. J. Acoust. Soc. Am. 106:1917-1924, 1999.

Wygonski J, Robert M. HEI Nucleus Research Interface (HEINRI) Specification. Internal Materials, 2002.

Xu L, Tsai Y, Pfingst BE. Features of stimulation affecting tonalspeech perception: implications for cochlear prostheses. J. Acoust. Soc. Am. 112:247-258, 2002.

Zeng FG, Turner CW. Binaural loudness matches in unilaterally impaired listeners. Q. J. Exp. Psychol. 43:565-583, 1991. 